\title{
Homogeneous Riemannian manifolds with a fixed isotropy representation
}

\author{
By Eduardo H. CATTANI* and L. N. MANN
}

(Received Dec. 15, 1977)

\section{Introduction.}

In this paper we give a classification of simply-connected homogeneous Riemannian manifolds $M=G / H$ where $H$ is isomorphic to a product of rotation groups and the linear isotropy representation of $H$ is a direct sum of standard representations with a trivial representation. This situation arises naturally in the study of homogeneous Riemannian manifolds which admit a large group of isometries. In fact if $M=I_{0}(M) / H$, where

$$
\operatorname{dim} I(M)>\frac{n^{2}}{4}+n, \quad n=\operatorname{dim} M \geqq 11
$$

then it follows that $H \cong S O(k) \times K$, with $k>n / 2, K \cong S O(n-k)$ and the linear isotropy representation of $H$ splits [5, Theorem 1.18].

Our results are quite simple to state if each of the rotation groups has order at least 3 . In that case $M$ is isometric to a product of a certain number of simply-connected manifolds of constant curvature together with a simplyconnected Lie group with a left-invariant metric. (Theorem B). If $H$ is isomorphic to a single rotation group, this appears to be consistent with some local results obtained by Kurita [8] a number of years ago. If some of the rotation groups in the decomposition of $H$ have order 2, then the description of the corresponding manifolds becomes more complicated. This is done in Section 4, where, in particular, we obtain a generalization of Cartan's classification [3] of 3-dimensional manifolds which admit a transitive group of motions of dimension 4.

In Section 5, we apply the above results to give an explicit description of those manifolds satisfying (1.1) and $n-3 \leqq k \leqq n$. This turns up some inaccuracies and extends some results in [7], while at the same time exhibiting the differences with the compact case studied by Lukesh. In [9] it is shown that if $M$ is compact and satisfies (1.1), then it must split isometrically with one factor being a standard sphere $S^{k}, k>n / 2$. As we shall see in Section 5, there

\footnotetext{
* Partially supported by NSF Grant MCS77-01735
} 
are uncountably many homogeneous metrics of strictly negative curvature on $\boldsymbol{R}^{n}$ with non-isomorphic isometry groups all of dimension $1 / 2\left(n^{2}-3 n+6\right)$.

\section{Algebraic preliminaries.}

$M=G / H$ will denote a connected homogeneous $n$-dimensional Riemannian manifold. Throughout this paper we shall assume that $G$ is connected and that the transitive action of $G$ on $M$ is effective. Let $g$ and $\mathfrak{h}$ denote the Lie algebras of $G$ and $H$ respectively. Since $H$ is compact we can choose a complementary subspace $\mathfrak{m}$ of $\mathfrak{h}$ in $\mathfrak{g}$ such that $[\mathfrak{h}, \mathfrak{m}] \cong \mathfrak{m}$. Moreover we can naturally identify $\mathfrak{m}$ with the tangent space of $M$ at the base point $z_{0}=\{H\} \in M$, and hence $\mathfrak{m}$ carries an inner product $\langle$,$\rangle induced by the Riemannian structure$ of $M$. If we let

then $\mathfrak{m}$ splits as

$$
\tilde{\mathfrak{n}}=\{X \in \mathfrak{m}:[\mathfrak{h}, X]=0\},
$$

$$
\mathfrak{m}=\mathfrak{m}_{1}+\mathfrak{\mathfrak { m }}
$$

where $\mathfrak{m}_{1}$ is the orthogonal complement of $\tilde{\mathfrak{m}}$ and therefore is $\operatorname{ad}(\mathfrak{G})$-invariant. '2.2) Proposition. Assume $H \cong S O(q), q \geqq 3$, and that the linear isotropy action of $H$ on $\mathfrak{m}_{1}$ is standard. Then

$$
\begin{aligned}
& {\left[\mathfrak{m}_{1}, \mathfrak{m}_{1}\right] \subseteq \mathfrak{h}} \\
& {\left[\tilde{\mathfrak{m}}, \mathfrak{m}_{1}\right] \subseteq \mathfrak{m}_{1} .}
\end{aligned}
$$

PRoof. We begin by showing that $\left[\mathfrak{m}_{1}, \mathfrak{m}_{1}\right]_{\tilde{\mathfrak{m}}}=0$, where $\left[\mathfrak{m}_{1}, \mathfrak{m}_{1}\right]_{\tilde{\mathfrak{m}}}$ denotes the projection of $\left[\mathfrak{m}_{1}, \mathfrak{m}_{1}\right]$ on $\tilde{\mathfrak{m}}$, relative to the decomposition $\mathfrak{g}=\mathfrak{h}+\mathfrak{m}_{1}+\tilde{\mathfrak{m}}$. Given $E_{1}, E_{2}$, orthonormal vectors in $\mathrm{m}_{1}$, let $A \in H$ be so that $A\left(E_{1}\right)=-E_{1}$, $A\left(E_{2}\right)=E_{2}$, where $A\left(E_{i}\right)$ denotes $\operatorname{Ad}(A) E_{i}$. Then $\left[E_{1}, E_{2}\right]_{\tilde{\mathfrak{m}}}=A\left(\left[E_{1}, E_{2}\right]_{\mathfrak{m}}\right)=$ $\left[A E_{1}, A E_{2}\right]_{\tilde{\mathfrak{m}}}=-\left[E_{1}, E_{2}\right]_{\tilde{\mathfrak{m}}}$ which implies $\left[E_{1}, E_{2}\right]_{\tilde{\mathfrak{m}}}=0$ and hence $\left[\mathfrak{m}_{1}, \mathfrak{m}_{1}\right]_{\tilde{\mathfrak{m}}}=0$.

In order to show that $\left[\mathfrak{m}_{1}, \mathfrak{m}_{1}\right]_{n_{1}}=0$, we choose an orthonormal basis $E_{1}, \cdots, E_{q}$ of $\mathfrak{m}_{1}$ and let

$$
\left[E_{i}, E_{j}\right]_{\mathfrak{H}_{1}}=\sum_{k=1}^{q} C_{i j}^{b} E_{k} .
$$

It is then enough to show that $C_{i j}^{k}=0$ for each $i, j, k$. If $q \geqq 4$, let $l \neq i, j, k$ and $A \in H$ the element defined by

We then have

$$
\begin{gathered}
A\left(E_{i}\right)=-E_{i}, \quad A\left(E_{l}\right)=-E_{l} \quad \text { and } \\
A\left(E_{s}\right)=E_{s} \quad \text { for } s \neq i, l .
\end{gathered}
$$

$$
\sum_{s=1}^{q} C_{i j}^{s} A\left(E_{s}\right)=A\left[E_{i}, E_{j}\right]_{m_{1}}=-\left[E_{i}, E_{j}\right]_{m_{1}}=-\sum_{s=1}^{q} C_{i j}^{s} E_{s}
$$


and comparing terms for $s=k$, this gives $C_{i j}^{k}=0$.

Suppose now that $q=3$. Let $E_{i}, 1 \leqq i \leqq 3$, be as before, and let $B \in H$ be defined by

$$
B\left(E_{1}\right)=-E_{1}, \quad B\left(E_{2}\right)=-E_{2}, \quad B\left(E_{3}\right)=E_{3} .
$$

Since $B\left(\left[E_{i}, E_{j}\right]_{\mathrm{m}_{1}}\right)=\left[B\left(E_{i}\right), B\left(E_{j}\right)\right]_{\mathrm{m}_{1}}$, it is straightforward to check that

$$
\left[E_{i}, E_{j}\right]_{\mathrm{n}_{1}}=\gamma_{k} E_{k}, \quad i \neq j \neq k \neq i, \quad \gamma_{k} \in \boldsymbol{R} .
$$

On the other hand for an arbitrary $A \in H$, let

$$
A\left(E_{i}\right)=\sum_{j=1}^{8} a_{j i} E_{j}
$$

We then have

$$
\gamma_{3} A\left(E_{3}\right)=A\left(\left[E_{1}, E_{2}\right]_{\mathrm{n}_{1}}\right)=\left[\sum_{j=1}^{3} a_{j_{1}} E_{j}, \sum_{k=1}^{3} a_{k 2} E_{k}\right]_{\mathrm{m}},
$$

and comparing the coefficients of $E_{3}$ we obtain

$$
\gamma_{3} a_{33}=\gamma_{3}\left(a_{11} a_{22}-a_{21} a_{12}\right)
$$

for every $A \in H$. It then follows that $\gamma_{3}=0$, and consequently $\left[E_{1}, E_{2}\right]_{\mathrm{ri}_{1}}=0$. This proves (2.3)

It remains to show $\left[\tilde{\mathfrak{m}}, \mathfrak{m}_{1}\right] \subseteq \mathfrak{m}_{1}$. Set

$$
\overline{\mathfrak{m}}_{1}=\left\{E \in \mathfrak{m}_{1}:[\tilde{\mathfrak{m}}, E]_{\tilde{\mathfrak{m}}}=0\right\} .
$$

Then $\overline{\mathfrak{m}}_{1}$ is an $\operatorname{Ad}(H)$-invariant subspace of $\mathfrak{m}_{1}$ and thus either $\overline{\mathfrak{m}}_{1}=\mathfrak{m}_{1}$ or $\overline{\mathfrak{m}}_{1}=\{0\}$. On the other hand it is easy to check that if $E \in \mathfrak{m}_{1}, A \in H$ are such that $A(E) \neq E$, then $E-A(E) \in \overline{\mathfrak{m}}_{1}$. Hence $\overline{\mathfrak{m}}_{1}=\mathfrak{m}_{1}$ and therefore $\left[\tilde{\mathfrak{m}}, \mathfrak{m}_{1}\right]_{\tilde{\mathfrak{m}}}=0$.

For each $X \in \tilde{\mathfrak{m}}$, let $\overline{\mathfrak{m}}_{1}(X)$ be the $\operatorname{Ad}(H)$-invariant subspace of $\mathfrak{m}_{1}$ defined by

$$
\overline{\mathfrak{m}}_{1}(X)=\left\{E \in \mathfrak{m}_{1}:[X, E]_{\mathfrak{h}}=0\right\} .
$$

If $\overline{\mathfrak{m}}_{1}(X)=\mathfrak{m}_{1}$ for all $X \in \tilde{\mathfrak{m}}$, then $\left[\tilde{\mathfrak{m}}, \mathfrak{m}_{1}\right]_{\mathfrak{r}}=0$ and (2.4) follows. Assume $\overline{\mathfrak{m}}_{1}(X)=0$ for some $X \in \tilde{\mathfrak{m}}$. This is clearly impossible if $q \geqq 4$ (or $q=2$ ) since $\left[X, \mathfrak{m}_{1}\right]_{\mathfrak{h}}$ would be a $q$-dimensional ideal of $\mathfrak{h} \cong s o(q)$. The case $q=3$ again requires a separate proof. Let $E_{i}, 1 \leqq i \leqq 3$, be as before and let $A_{i j}, 1 \leqq i \neq j \leqq 3$, be elements of $\mathfrak{h} \cong s o(3)$ defined by

$$
\begin{aligned}
& {\left[A_{i j}, E_{i}\right]=-E_{j}, \quad\left[A_{i j}, E_{j}\right]=E_{i}} \\
& {\left[A_{i j}, E_{k}\right]=0, \quad k \neq i, j .}
\end{aligned}
$$

Clearly $\left\{A_{i j}, 1 \leqq i<j \leqq 3\right\}$ is a basis of $\mathfrak{h}$ and $A_{i j}=-A_{j i}$. One can readily check that

$$
\left[A_{i j}, A_{j k}\right]=A_{i k}
$$


Let

Since

$$
\left[X, E_{i}\right]_{\mathfrak{h}}=\alpha_{i} A_{12}+\beta_{i} A_{13}+\gamma_{i} A_{23} .
$$

$$
\begin{aligned}
& {\left[A_{23},\left[X, E_{1}\right]_{\mathfrak{h}}\right]=\left[A_{23},\left[X, E_{1}\right]\right]_{\mathfrak{h}}} \\
& =\left[\left[A_{23}, X\right], E_{1}\right]_{\mathfrak{h}}+\left[X,\left[A_{23}, E_{1}\right]_{\mathfrak{h}}=0,\right.
\end{aligned}
$$

using (2.5) and (2.6) we obtain

$$
0=\left[A_{23}, \alpha_{1} A_{12}+\beta_{1} A_{13}+\gamma_{1} A_{23}\right]=-\alpha_{1} A_{13}+\beta_{1} A_{12}
$$

which implies $\alpha_{1}=\beta_{1}=0$. Similarly one can show that $\alpha_{2}=\gamma_{2}=\beta_{3}=\gamma_{3}=0$ and $\alpha_{3}=-\beta_{2}=\gamma_{1}=\lambda$.

On the other hand, since by $(2.3),\left[\mathfrak{m}_{1}, \mathfrak{m}_{1}\right] \subseteq \mathfrak{h}$ we get

$$
\begin{aligned}
0 & =\left[X,\left[E_{1}, E_{3}\right]_{\mathrm{r}_{1}}=\left[\left[X, E_{1}\right], E_{3}\right]_{\mathrm{m}_{1}}+\left[E_{1},\left[X, E_{3}\right]\right]_{\mathrm{m}_{1}}\right. \\
& =\left[\left[X, E_{1}\right]_{\mathfrak{h}}, E_{3}\right]+\left[E_{1},\left[X, E_{3}\right]_{\mathfrak{\eta}}\right] \\
& =\left[\lambda A_{23}, E_{3}\right]+\left[E_{1}, \lambda A_{12}\right]=\lambda E_{2}+\lambda E_{2}=2 \lambda E_{2} .
\end{aligned}
$$

Hence $\lambda=0$ and $\left[\tilde{\mathfrak{m}}, \mathfrak{m}_{1}\right] \subseteq \mathfrak{m}_{1}$. This completes the proof of Proposition (2.2).

For each $X \in \tilde{\mathfrak{m}}$, the linear transformation

$$
\operatorname{ad}(X): \mathfrak{m}_{1} \rightarrow \mathfrak{m}_{1}
$$

commutes with the standard action of $H \cong S O(q)$ on $\mathfrak{m}_{1}$. Hence there exists a linear functional $\alpha \in \tilde{\mathfrak{m}}^{*}$, such that

$$
[X, E]=\alpha(X) E, \quad X \in \tilde{\mathfrak{m}}, \quad E \in \mathfrak{m}_{1} .
$$

We set

$$
\tilde{\mathfrak{m}}^{\prime}=\operatorname{ker} \alpha=\left\{X \in \tilde{\mathfrak{m}}:\left[X, \mathfrak{m}_{1}\right]=0\right\}
$$

Then, either $\tilde{\mathfrak{m}}=\tilde{\mathfrak{m}}^{\prime}$ or $\operatorname{dim} \tilde{\mathfrak{m}}^{\prime}=\operatorname{dim} \tilde{\mathfrak{m}}-1$.

(2.9) Proposition. ĩ is a subalgebra of $\mathfrak{g}$ and $\tilde{\mathfrak{m}}^{\prime}$ is an ideal in $\mathfrak{g}$.

Proof. First of all we notice that $[\tilde{\mathfrak{m}}, \tilde{\mathfrak{m}}]_{\mathrm{m}_{1}}=0$ since $[\tilde{\mathfrak{m}}, \tilde{\mathfrak{m}}]_{\mathfrak{r}_{1}}$ would be a subspace of $\mathfrak{m}_{1}$ where $\mathfrak{h}$ acts trivially. Similarly we have

$$
\left[\mathfrak{h},[\tilde{\mathfrak{m}}, \tilde{\mathfrak{m}}]_{\mathfrak{h}}\right]=0
$$

which implies $[\tilde{\mathfrak{m}}, \tilde{\mathfrak{m}}]_{\mathfrak{h}}=0$ since $\mathfrak{h} \cong s o(q)$ contains no non-trivial abelian ideals for $q \geqq 3$. This proves the first statement in (2.9).

Let $X_{1}, X_{2} \in \tilde{\mathfrak{m}}, E \in \mathfrak{m}_{1}$. Then

$$
\left[\left[X_{1}, X_{2}\right], E\right]=\left(\alpha\left(X_{1}\right) \alpha\left(X_{2}\right)-\alpha\left(X_{2}\right) \alpha\left(X_{1}\right)\right) E=0 .
$$

This implies $[\tilde{\mathfrak{m}}, \tilde{\mathfrak{m}}] \subseteq \tilde{\mathfrak{m}}^{\prime}$ and since $\left[\mathfrak{h}+\mathfrak{m}_{1}, \tilde{\mathfrak{m}}^{\prime}\right]=0$, the proposition follows.

(2.10) COROLlaRY. If $\tilde{\mathfrak{m}}^{\prime}=\tilde{\mathfrak{m}}$ then the decomposition 
is a direct sum of ideals.

$$
\mathfrak{g}=\left(\mathfrak{h}+\mathfrak{m}_{1}\right) \oplus \tilde{\mathfrak{m}}
$$

(2.11) LEMMA. If $\tilde{\mathfrak{m}}^{\prime} \neq \tilde{\mathfrak{m}}$, then $\mathfrak{m}_{1}$ is an abelian ideal in $\mathrm{g}$.

Proof. Using (2.2) it is enough to show that $\left[\mathfrak{m}_{1}, \mathfrak{m}_{1}\right]_{\mathfrak{h}}=0$. Let $E_{1}, E_{2} \in \mathfrak{m}_{1}$ and choose $X \in \tilde{\mathfrak{m}}$ such that $\alpha(X)=1$. Then

$$
\begin{aligned}
0 & =\left[X,\left[E_{1}, E_{2}\right]_{\mathfrak{h}}\right]=\left[X,\left[E_{1}, E_{2}\right]\right]_{\mathfrak{h}} \\
& =\left[\alpha(X) E_{1}, E_{2}\right]+\left[E_{1}, \alpha(X) E_{2}\right]=2\left[E_{1}, E_{2}\right]
\end{aligned}
$$

and the result follows.

(2.12) Proposition. If $\tilde{\mathfrak{m}}^{\prime} \neq \tilde{\mathfrak{m}}$, then $\mathfrak{m}=\mathfrak{m}_{1}+\tilde{\mathfrak{m}}$ is an ideal in $\mathfrak{g}$.

Proof. This is a consequence of Proposition (2.2) and the above lemma.

\section{Global results.}

Throughout this section $M$ will denote a connected and simply-connected homogeneous Riemannian manifold $M=G / H$, with $G$ a connected subgroup of $I(M)$ acting effectively on $M$. We assume further that

$$
H \cong H_{1} \times \cdots \times H_{k}
$$

and the linear isotropy representation of $H$ splits. The Lie algebra $g$ of $G$ has therefore a decomposition

$$
\mathfrak{g}=\mathfrak{h}_{1}+\cdots+\mathfrak{h}_{k}+\mathfrak{m}_{1}+\cdots+\mathfrak{m}_{k}+\tilde{\mathfrak{m}}
$$

where $\mathfrak{h}_{i}$ leaves $\mathfrak{m}_{i}$ invariant and acts trivially on $\tilde{\mathfrak{m}}$ and $\mathfrak{m}_{j}, j \neq i$. It is clear that the decomposition $\mathfrak{m}=\mathfrak{m}_{1}+\cdots+\mathfrak{m}_{k}+\tilde{\mathfrak{m}}$ is orthogonal relative to the inner product induced in $\mathfrak{m}$ by the Riemannian structure of $M$.

(3.2) LeMmA. Let $M=G /\left(H_{1} \times H_{2}\right)$ be as above, $\mathfrak{g}=\mathfrak{h}_{1}+\mathfrak{h}_{2}+\mathfrak{m}_{1}+\mathfrak{m}_{2}+\tilde{\mathfrak{m}}$ as in (3.1). If $\mathfrak{g}=\mathfrak{h}_{2}+\mathfrak{m}_{1}+\mathfrak{m}_{2}+\tilde{\mathfrak{m}}$ is an ideal of $\mathfrak{g}$ and $S$ denotes the corresponding analytic subgroup of $G$, then $M=S / H_{2}$.

Proof. The subgroup $S$ acts as an effective group of isometries of $M$. Since $\mathfrak{Z}_{\cap} \cap\left(\mathfrak{h}_{1}+\mathfrak{h}_{2}\right)=\mathfrak{h}_{2}$, and $S$ is normal in $G$, we have for any $z \in M$

$$
\begin{aligned}
\operatorname{dim} S_{z} & =\operatorname{dim}\left(S \cap G_{z_{0}}\right)=\operatorname{dim}\left(S \cap\left(H_{1} \times H_{2}\right)\right) \\
& =\operatorname{dim} \mathfrak{h}_{2},
\end{aligned}
$$

where $z_{0}=\{H\}$ is the base point in $M$. Hence for any $z \in M$, the orbit $S(z)$ has the same dimension as $M$ and is therefore open in $M$, and consequently every orbit is also closed. Since $M$ is assumed to be connected this proves (3.2).

In particular if $H_{2}=\{e\}, \xi=\mathfrak{m}$ and we obtain the following standard result. (3.3) Lemma. Let $M=G / H$ be as above and assume that the Lie algebra $g$ of 
$G$ admits a decomposition $\mathfrak{g}=\mathfrak{h}+\mathfrak{m}$, where $\mathfrak{h}$ is the Lie algebra of $H$ and $\mathfrak{m}$ is an ideal of $\mathrm{g}$. Then $M$ is isometric to a Lie group with a left-invariant metric.

We shall also need the following:

(3.4) LEMMA. Let $M=G /\left(H_{1} \times H_{2}\right)$ be a connected simply-connected, homogeneous Riemannian manifold. Assume further that the decomposition (3.1)

$$
\mathfrak{g}=\left(\mathfrak{h}_{1}+\mathfrak{m}_{1}\right) \oplus\left(\mathfrak{h}_{2}+\mathfrak{m}_{2}+\tilde{\mathfrak{m}}\right)=\mathfrak{g}_{1} \oplus \mathfrak{g}_{2}
$$

is a direct sum of ideals. Then there exists closed connected normal subgroups $G_{i}, \quad i=1,2$ of $G$ with Lie algebras $\mathfrak{g}_{i}, i=1,2$, such that $M$ is isometric to the product $M_{1} \times M_{2}$ where $M_{i}$ is the simply-connected homogeneous Riemannian manifold $G_{i} / H_{i}$.

Proof. Let $\hat{G}$ denote the universal covering group of $G$, and $\hat{G}_{i}, i=1,2$, the analytical subgroups of $\hat{G}$ with Lie algebras $g_{i}, i=1,2$, respectively. Then $\hat{G}_{i}$ is a simply-connected closed normal subgroup of $\hat{G}$. Since $\mathfrak{h}_{i} \leqq \mathfrak{g}_{i}$, let $\hat{H}_{i} \cong \hat{G}_{i}$ be the corresponding connected subgroup. Now $\hat{G}$ acts as a transitive group of isometries (although possibly not effectively) on $M$. So

$$
\hat{M}=\hat{G} / G_{z_{0}}
$$

where $\hat{G}_{z_{0}}$ is the isotropy subgroup at the base point $z_{0} \in M$. It is clear however that

$$
\hat{G}_{z_{0}}=\hat{H}_{1} \times \hat{H}_{2}
$$

since they are connected subgroups with the same Lie algebra. Therefore $M$ splits diffeomorphically as

$$
M=\hat{G}_{1} / \hat{H}_{1} \times \hat{G}_{2} / \hat{H}_{2} .
$$

Let $\pi: \hat{G} \rightarrow G$ be the natural projection, $G_{i}=\pi\left(\hat{G}_{i}\right), H_{i}$ as before. The subgroup $N=\operatorname{Ker} \pi$ is normal in $\hat{G}$ and is contained in $\hat{G}_{z_{0}}$. Moreover since $N$ acts trivially on $M, N_{i}=\hat{G}_{i} \cap N$ acts trivially on $M_{i}=\hat{G}_{i} / \hat{H}_{i}$, hence

$$
M_{i}=\hat{G}_{i} / \hat{H}_{i}=\frac{\hat{G}_{i} /\left(\hat{G}_{i} \cap N\right)}{\hat{H}_{i} /\left(\hat{H}_{i} \cap N\right)}=G_{i} / H_{i}^{*} .
$$

But $H_{i}$ and $H_{i}^{*}$ are both connected and have the same Lie algebra $\mathfrak{h}_{i}$. Therefore $H_{i}^{*}=H_{i}$ and $M_{i}=G_{i} / H_{i}$.

It remains to show that (3.5) is an isometric splitting, or equivalently, that for any $z=\left(z_{1}, z_{2}\right) \in M$, the subspaces $T_{z_{1}}\left(M_{1}\right)$ and $T_{z_{2}}\left(M_{2}\right)$ are orthogonal with respect to the Riemannian inner product in $T_{z}(M)$. But this is clear at the base point $z_{0}=\left(z_{1}^{0}, z_{2}^{0}\right)$ since $T_{z_{1}^{0}}\left(M_{1}\right) \cong \mathfrak{m}_{1}$ and $T_{z_{2}^{0}}\left(M_{2}\right) \cong \mathfrak{m}_{2}+\tilde{\mathfrak{m}}$, and at any other point by homogeneity.

In what follows, $\rho_{q}$ and $\theta_{k}$ will denote the standard and trivial representations of $S O(q)$ on $\boldsymbol{R}^{q}$ and $\boldsymbol{R}^{k}$ respectively. 
TheOrem A. Suppose $M=G / H$ is a connected simply-connected n-dimensional homogeneous Riemannian manifold. If $H$ is isomorphic to $S O(q), 3 \leqq q \leqq n$, and the linear isotropy representation of $H$ is $\rho_{q} \oplus \theta_{n-q}$, then either

(1) $M$ is isometric to $M_{1}^{(q)} \times M_{2}^{(n-q)}$ where $M_{1}$ is a q-dimensional simply-connected space of constant curvature and $M_{2}$ is isometric to an $(n-q)$-dimensional simply-connected Lie group with a left-invariant metric. Furthermore $G \cong I_{0}\left(M_{1}\right)$ $\times M_{2}$, where $I_{0}\left(M_{1}\right)$ is the identity connected component of the full group of isometries of $M_{1}$, or

(2) $M$ is isometric to a Lie group with a left-invariant metric and $G$ is isomorphic to a semi-direct product of $S O(q)$ with $M$.

Proof. Let $\mathfrak{g}=\mathfrak{h}+\mathfrak{m}_{1}+\tilde{\mathfrak{m}}$ be as in (2.1). If $\tilde{\mathfrak{m}}^{\prime}=\tilde{\mathfrak{m}}$, then by Corollary (2.10)

$$
\mathfrak{g}=\left(\mathfrak{h}+\mathfrak{m}_{1}\right) \oplus \tilde{\mathfrak{m}}
$$

is a direct sum of ideals. Applying lemma (3.4) we can conclude that $M$ splits isometrically

$$
M=G_{1} / S O(q) \times G_{2} .
$$

But $G_{1}$ acts effectively on the $q$-dimensional manifold $M_{1}$ and $\operatorname{dim} G_{1}=\frac{1}{2} q(q+1)$, hence $M_{1}$ has constant curvature. We thus obtain (1).

If $\tilde{\mathfrak{m}}^{\prime} \neq \tilde{\mathfrak{m}}$, then by (2.12) $\mathfrak{m}$ is an ideal in $\mathfrak{g}$. Lemma (3.3) now applies to give (2).

(3.6) REMARK. In (2) of Theorem A, observe that by (2.11) $\mathfrak{m}_{1}$ is an abelian ideal of $\mathrm{g}$, and hence the Lie group $M$ contains a simply-connected closed normal abelian subgroup of dimension $q$. It then follows that if $M$ is compact we must have $\tilde{\mathfrak{m}}^{\prime}=\tilde{\mathfrak{m}}$ and thus case (1) in Theorem A. This is the case studied by Lukesh [9].

Also, since by (2.9) $\tilde{\mathfrak{m}}^{\prime}$ is an ideal in $\mathfrak{g}$, the corresponding analytic subgroup $K$ of $G$ is normal. Moreover $K$ coincides with the identity connected component of the centralizer of $\mathfrak{h}+\mathfrak{m}_{1}$ in $G$, and is therefore closed. One can easily check that $K \cap H=\{e\}$, from which it follows, since $K$ is normal, that $K$ acts freely on $M$. Moreover the orbit space $M / K$ with its induced metric can be seen to be a space of constant negative curvature [6, Theorem 3.3], hence diffeomorphic to Euclidean space. Since $M$ is a principal fiber bundle over $M / K, M$ is diffeomorphic to the product of $K$ with a Euclidean space.

THEOREM B. Suppose $M=G / H$, is a connected simply-connected n-dimensional homogeneous Riemannian manifold. If $H$ is isomorphic to a product $S O\left(q_{1}\right) \times$ $S O\left(q_{2}\right) \times \cdots \times S O\left(q_{k}\right)$ where

$$
q_{i} \geqq 3 \text { for all } i \text { and } \sum_{i=1}^{k} q_{i} \leqq n
$$

and if the linear isotropy representation of $H$ splits as 


$$
\rho_{q_{1}} \oplus \rho_{q_{2}} \oplus \cdots \oplus \rho_{q_{k}} \oplus \theta_{n-\Sigma q_{i}},
$$

then there exists some subset $q_{i_{1}}, \cdots, q_{i_{l}}$ of the $q_{i}$ 's such that $M$ is isometric to

$$
M_{1} \times M_{2} \times \cdots \times M_{l} \times M_{l+1}
$$

where $M_{j}, 1 \leqq j \leqq l$, is a $q_{i_{j}}$-dimensional simply-connected manifold of constant curvature and $M_{l+1}$ is an $\left(n-\sum_{j=1}^{l} q_{i_{j}}\right)$-dimensional simply-connected Lie group with a left-invariant metric.

Proof. We decompose $g$ according to (3.1) as

Let

$$
\mathrm{g}=\mathfrak{h}_{1}+\cdots+\mathfrak{h}_{k}+\mathfrak{m}_{1}+\cdots+\mathfrak{m}_{k}+\tilde{\mathfrak{m}} .
$$

$$
\mathfrak{g}=\mathfrak{h}_{2}+\cdots+\mathfrak{h}_{k}+\mathfrak{m}_{1}+\cdots+\mathfrak{m}_{k}+\mathfrak{m}
$$$$
\xi_{1}=\mathfrak{m}_{1}
$$$$
\tilde{\Xi}=\mathfrak{h}_{2}+\cdots+\mathfrak{h}_{k}+\mathfrak{m}_{2}+\cdots+\mathfrak{m}_{k}+\tilde{\mathfrak{m}}
$$

and observe that $\left[\mathfrak{h}_{1}, \tilde{\tilde{s}}\right]=0$. Then

$$
g=\mathfrak{h}_{1}+\mathfrak{g}_{1}+\tilde{\xi}
$$

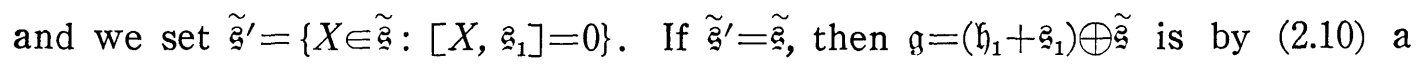
direct sum of ideals. It follows then from Lemma (3.4) that $M$ is isometric to $M_{1} \times M^{*}$ where $M_{1}$ is a $q_{1}$-dimensional simply-connected manifold of constant curvature and $M^{*}=G_{2} /\left(H_{2} \times \cdots \times H_{k}\right)$, where $G_{2}$ is the analytic subgroup of $G$ with Lie algebra $\tilde{\varrho}$. We proceed inductively on $M^{*}$ with respect to $k$.

If $\widetilde{\mathfrak{Z}}^{\prime} \neq \tilde{\mathfrak{z}}$, then $\tilde{z}=\tilde{z}_{1}+\widetilde{\mathfrak{z}}$ is an ideal of $\mathrm{g}$. Lemma (3.2) now implies that $M=S /\left(H_{2} \times \cdots \times H_{k}\right)$, where $S$ is the analytic subgroup corresponding to 2 . Again an inductive process completes the proof.

(3.7) REMARK. Using the results of Section 2, it is possible to give rather explicit descriptions of the Lie algebra $g$ and the group $G$. Although in the general case this is not particularly enlightening, we will do it in Section 5 for some special cases.

We end this section with the following result, a local version of which is due to Wakakuwa [11, Theorem 2].

THEOREM C. (Wakakuwa). Suppose $M=G / H$ is a connected simply-connected $n$-dimensional homogeneous Riemannian manifold. Assume that $H \cong H_{1} \times \cdots \times H_{k}$ and the linear isotropy representation of $H$ is faithful and splits. Then $M$ is isometric to a product

$$
M \cong M_{1} \times \cdots \times M_{k},
$$

where $M_{i}=G_{i} / H_{i}$ for $G_{i}$ some connected normal subgroup of $G$.

Proof. We sketch a proof using the techniques of Section 2. Infinitesi- 
mally we have

$$
\mathfrak{g}=\mathfrak{h}_{1}+\cdots+\mathfrak{h}_{k}+\mathfrak{m}_{1}+\cdots+\mathfrak{m}_{k} .
$$

It can be shown that $\mathfrak{g}_{i}=\mathfrak{h}_{i}+\mathfrak{m}_{i}$ is an ideal of $\mathfrak{g}$ for $1 \leqq i \leqq k$, and therefore $\mathfrak{g}$ splits as $\mathfrak{g}=\mathrm{g}_{1} \oplus \cdots \oplus \mathfrak{g}_{k}$. The result now follows inductively from Lemma (3.4).

\section{Rotation groups of order 2 .}

In this section we will study Riemannian homogeneous spaces of the form $G / H$, where $H \cong S O(2)$ and the linear isotropy representation of $H$ is equivalent to $\rho_{2} \oplus \theta_{n-2}$. In particular, in the case $\operatorname{dim} M=3$ we will recover Cartan's classification [3] of 3-dimensional manifolds admitting a transitive group of isometries of dimension 4. The general case where $H$ is isomorphic to a product of rotation groups, some of which are of order 2, can be treated along the same lines as Theorem B of the preceding section. In Section 5 we shall study one such case in detail.

Keeping the notation of Section 2 we can write

$$
\mathfrak{g}=\mathfrak{h}+\mathfrak{m}_{1}+\tilde{\mathfrak{m}}
$$

where $[\mathfrak{h}, \tilde{\mathfrak{m}}]=0, \operatorname{dim} \mathfrak{m}_{1}=2, \mathfrak{h} \cong s o(2)$ acts on $\mathfrak{m}_{1}$ in the natural way and $\mathfrak{m}_{1}$ is orthogonal to $\mathfrak{\mathfrak { m }}$ relative to the natural inner product in $\mathfrak{m}=\mathfrak{m}_{1}+\mathfrak{\mathfrak { m }}$. It is easy to check that (2.4) is still valid in this case, that is

$$
\left[\tilde{\mathfrak{m}}, \mathfrak{m}_{1}\right] \leqq \mathfrak{m}_{1} .
$$

Let now $E_{1}, E_{2}$ be an orthonormal basis of $\mathfrak{m}_{1}$ and $H_{0}$ the element of $\mathfrak{h}$ defined by

$$
\left[H_{0}, E_{1}\right]=-E_{2}, \quad\left[H_{0}, E_{2}\right]=E_{1} .
$$

Given $X \in \tilde{\mathfrak{m}}$ we can write

$$
\left[X, E_{i}\right]=\sum_{j=1}^{2} a_{i j} E_{j}, \quad i=1,2 .
$$

Since $\left[H_{0},\left[X, E_{1}\right]\right]=-\left[X, E_{2}\right]$ we deduce that $a_{11}=a_{22}$ and $a_{12}=-a_{21}$. Hence there exist linear functionals $\alpha, \beta \in \tilde{\mathfrak{m}}^{*}$ such that

$$
\begin{aligned}
& {\left[X, E_{1}\right]=\alpha(X) E_{1}-\beta(X) E_{2}} \\
& {\left[X, E_{2}\right]=\beta(X) E_{1}+\alpha(X) E_{2} .}
\end{aligned}
$$

Let $X_{1}, \cdots, X_{n}$ be an orthonormal basis of $\tilde{\mathfrak{m}}$ such that $\beta\left(X_{i}\right)=0$ for $1 \leqq i \leqq n-1$ and let $\beta\left(X_{n}\right)=b$. Then replacing $\tilde{\mathfrak{m}}$ by the subspace spanned by $X_{1}, \cdots, X_{n-1}$, $X_{n}-b H_{0}$, and making the corresponding change in $\mathfrak{n}$, we can assume that

$$
[X, E]=\alpha(X) E, \quad X \in \tilde{\mathfrak{m}}, \quad E \in \mathfrak{m}_{1} .
$$


(4.5) LemMA. $\tilde{\mathfrak{m}}$ is a subalgebra of $\mathfrak{g}$ and $\tilde{\mathfrak{m}}^{\prime}=\operatorname{Ker} \alpha \leqq \tilde{\mathfrak{m}}$ is an ideal in $\mathfrak{g}$.

Proof. As in (2.9) we have $[\tilde{\mathfrak{m}}, \tilde{\mathfrak{m}}]_{\mathfrak{n}_{1}}=0$. On the other hand, let $X_{i} \in \tilde{\mathfrak{m}}$, $i=1,2$, and set

Then

$$
\left[X_{1}, X_{2}\right]_{\mathfrak{h}}=a H_{0} .
$$

$$
\begin{aligned}
0 & =\left[\left[X_{1}, X_{2}\right], E_{1}\right]=a\left[H_{0}, E_{1}\right]+\left[\left[X_{1}, X_{2}\right]_{\mathfrak{m}}, E_{1}\right] \\
& =-a E_{2}+\alpha\left(\left[X_{1}, X_{2}\right]_{\mathfrak{m}}\right) E_{1} .
\end{aligned}
$$

Hence $a=0$ and thus $[\tilde{\mathfrak{m}}, \tilde{\mathfrak{m}}] \subseteq \tilde{\mathfrak{m}}$. The second statement follows as in (2.9).

As in the proof of Proposition (2.2) one can show that

$$
\left[\mathfrak{m}_{1}, \mathfrak{m}_{1}\right]_{\mathfrak{n}_{1}}=0 \text {. }
$$

However, it is not true in general that $\left[\mathfrak{m}_{1}, \mathfrak{m}_{1}\right]_{\tilde{\mathfrak{m}}}=0$. This is what distinguishes this case from the one discussed in Section 2.

(4.7) THEOREM. Let $M=G / H$ be a connected simply-connected n-dimensional homogeneous Riemannian manifold. Assume $H \cong S O(2)$ and the linear isotropy representation of $H$ is equivalent to $\rho_{2} \oplus \theta_{n-2}$. Then $M$ is one of the following:

(1) $M$ is isometric to a product $M=M_{1}^{(2)} \times M_{2}^{(n-2)}$ where $M_{1}$ is a 2-dimensional simply-connected space of constant curvature and $M_{2}$ is a simply-connected Lie group with a left-invariant metric. Moreover $G \cong I_{0}\left(M_{1}\right) \times M_{2}$.

(2) $M$ is isometric to a simply-connected Lie group with a left-invariant metric and $G$ is isomorphic to a semi-direct product of $S O(2)$ with $M$.

(3) $M$ is a principal fiber bundle, with abelian structural group, over the product of a 2-dimensional space with non-zero constant curvature and a simplyconnected Lie group with a left-invariant metric.

Proof. We begin by considering the case $\tilde{\mathfrak{m}}^{\prime}=\tilde{\mathfrak{m}}$, that is $\left[\tilde{\mathfrak{m}}, \mathfrak{m}_{1}\right]=0$. Let $z(\mathfrak{g}), z(\tilde{\mathfrak{m}})$ denote the centers of $g$ and $\tilde{\mathfrak{m}}$ respectively. Then

$$
z(g)=z(\tilde{\mathfrak{m}}) \text {. }
$$

In fact, let $Z \in \mathfrak{z}(\mathrm{g})$. If we decompose $Z$ according to [4.1) as $Z=Z_{\mathfrak{\xi}}+Z_{\mathrm{m}_{1}}+Z_{\mathfrak{m}}$, then since $Z_{\mathfrak{\natural}}$ acts trivially on $\mathfrak{m}_{1}$ we must have $Z_{\mathfrak{h}}=0$. Similarly $Z_{\mathfrak{m}_{1}}$ defines a subspace of $\mathfrak{m}_{1}$ where $\mathfrak{h}$ acts trivially, hence $Z_{\mathfrak{m}_{1}}=0$ and $\mathfrak{z}(\mathfrak{g}) \subseteq \tilde{\mathfrak{m}}$. Since clearly $z(\tilde{\mathfrak{m}}) \cong z(\mathfrak{g})$ we obtain $(4.8)$.

Let $E_{i}, i=1,2$, be an orthonormal basis of $\mathfrak{m}_{1}$ and set

$$
\left[E_{1}, E_{2}\right]=\lambda H_{0}+\mu Z, \quad \mu \geqq 0,
$$

where $Z \in \tilde{\mathfrak{m}}$ is a unit vector. Notice that for $\mu \neq 0, Z \in z(\tilde{\mathfrak{m}})=z(\mathfrak{g})$. If $\mu=0$, then the decomposition

$$
\mathfrak{g}=\left(\mathfrak{h} \oplus \mathfrak{m}_{1}\right) \oplus \tilde{\mathfrak{m}}=\mathfrak{g}_{\lambda} \oplus \tilde{\mathfrak{m}},
$$

where $g_{\lambda}$ is the 3-dimensional Lie algebra $\left\{H_{0}, E_{1}, E_{2}:\left[H_{0}, E_{1}\right]=-E_{2},\left[H_{0}, E_{2}\right]\right.$ 
$\left.=E_{1},\left[E_{1}, E_{2}\right]=\lambda H_{0}\right\}$, is a direct sum of ideals and, consequently, it follows from Lemma (3.4) that $M$ splits isometrically as $M_{1}^{(2)} \times M_{2}^{(n-2)}$. Moreover $M_{1}$ is a 2-dimensional space of constant curvature, positive if $\lambda<0$, negative if $\lambda>0$ and zero if $\lambda=0$. This gives (1) in (4.7).

If $\mu \neq 0, \lambda=0$; then $\mathfrak{m}$ is an ideal in $\mathfrak{g}$ and applying Lemma (3.3) we obtain case (2).

Assume now that $\lambda \neq 0, \mu \neq 0$. Let $g_{1}=\mathfrak{g} / \mathfrak{z}(\mathfrak{g})$. Then $\mathfrak{g}_{1}$ is a split Lie algebra :

$$
\mathrm{g}_{1} \cong \mathrm{g}_{\lambda} \oplus \mathbb{R} \text {. }
$$

If $C$ denotes the analytic subgroup of $G$ whose Lie algebra is $z(g)$, then $C$ is closed and normal. Moreover $C$ acts freely on $M$ since $C \cap H=\{e\}$. Therefore

$$
C \rightarrow M \rightarrow M / C
$$

is a principal fiber bundle. The group $G / C$ acts as an effective group of isometries on $M / C$, and the isotropy subgroup at any point is isomorphic to $S O(2)$. It follows then from (4.9) that $M / C$ is as in (1) of (4.7). We thus obtain case (3).

Finally, suppose $\tilde{\mathfrak{m}}^{\prime} \neq \tilde{\mathfrak{m}}$. Let $X \in \tilde{\mathfrak{m}}$ be a unit vector such that $\alpha(X)=a>0$. As in (2.11) we have $\left[\mathfrak{m}_{1}, \mathfrak{m}_{1}\right]_{\mathfrak{h}}=0$ and in fact $\left[\mathfrak{m}_{1}, \mathfrak{m}_{1}\right] \subseteq \tilde{\mathfrak{m}}^{\prime}$. Therefore $\mathfrak{m}$ is an ideal in $g$ and using (3.3) we obtain case (2) again. Notice that $\tilde{\mathfrak{m}}^{\prime}$ is an ideal in $\mathfrak{m}$ and $\mathfrak{m} / \tilde{\mathfrak{m}}^{\prime}$ is isomorphic to the 3 -dimensional Lie algebra

$$
\left\{X, E_{1}, E_{2}:\left[X, E_{i}\right]=a E_{i},\left[E_{1}, E_{2}\right]=0\right\} .
$$

(4.11) Example. Suppose $\operatorname{dim} M=3$, hence $\operatorname{dim} G=4$ and we are in the situation studied by E. Cartan in [3]. Case (1) Theorem (4.7) gives, of course, an isometric product of a 2-dimensional space of constant curvature and a line. In case (2) we have two possibilities depending upon whether $\tilde{\mathfrak{m}}^{\prime}=\tilde{\mathfrak{m}}$ or $\tilde{\mathfrak{m}}^{\prime} \neq \tilde{\mathfrak{m}}$. In the first situation $M$ is isometric to the Heisenberg group (strictly upper triangular $3 \times 3$-matrices), endowed with a left-invariant metric, while in the second $M$ is a solvable Lie group whose Lie algebra is described by (4.10). Moreover, with respect to any left-invariant metric $M$ will have strictly negative curvature [4].

The most interesting case is that described in (3) of (4.7). If $\lambda>0$, then $M$ is a principal fiber bundle over a space of constant negative curvature and hence it is diffeomorphic to Euclidean 3-space. If $\lambda<0$, however, one can check that for $\mu^{2}=-\lambda, M$ has constant positive curvature and is, therefore, diffeomorphic to $S^{3}$. Since a change in the metric in the direction of $Z$ allows us to change the value of $\mu$ arbitrarily we see that if $\lambda<0$ then $M$ is diffeomorphic to $S^{3}$ although not, in general, isometric. 
(4.12) REMARK. In the case $\operatorname{dim} M=4$, the results in this section complete the classification in [7], where the cases $\tilde{\mathfrak{m}}^{\prime}=\tilde{\mathfrak{m}}$ and $\mu=0$ are treated.

\section{Some special cases.}

In this section we apply the preceding results to give a classification of the $n$-dimensional connected, simply-connected homogeneous Riemannian manifolds $M=G / H$ where $H \cong S O(k) \times K, n-3 \leqq k \leqq n$ and such that the linear isotropy representation of $H$ is standard. As is well-known, if $k=n$ then $M$ is a space of constant curvature; while if $k=n-1$ then $M$ is either an $n$-dimensional space of constant negative curvature or a product of an $(n-1)$ dimensional space of constant curvature and a line [6].

If $k=n-2$ and $K=S O(2)$ it follows from Theorem $C$ that $M$ is isometrically equivalent to a product $M=M_{1}^{(n-2)} \times M_{2}^{(2)}$ of simply-connected spaces of constant curvature. The case $K=\{e\}$ has been studied by Kobayashi and Nagano in [7]; however their results turn out to be valid only under the additional assumption that $M$ be naturally reductive. When this restriction is removed one obtains a one-parameter family of new examples. The case $H=S O(2)$ has been studied in Section 4 ; for $n-2 \geqq 3$ we have

(5.1) THEOREM. Let $M=G / H$ be a simply-connected $n$-dimensional homogeneous Riemannian manifold and assume that $H \cong S O(n-2), n-2 \geqq 3$ and the linear isotropy representation of $H$ is the standard one. Then $M$ is one of the following :

(1) $M$ is isometric to a product $M_{1}^{(n-2)} \times M_{2}^{(2)}$ where $M_{1}$ is a simply-connected $(n-2)$-dimensional space of constant curvature and $M_{2}$ is a simply-connected Lie group with a left-invariant metric. Moreover $G \cong I_{0}\left(M_{1}\right) \times M_{2}$.

(2) $M \cong M_{1}^{(n-1)} \times \boldsymbol{R}$, where $M_{1}$ is a space of constant negative curvature and $G \cong G_{1} \times \boldsymbol{R}$, where the Lie algebra $\mathfrak{g}_{1}$ of $G_{1}$ is the one described by Kobayashi in [6, Theorem 3.3].

(3) $M$ is isometric to a solvable Lie group $M(\lambda), \lambda \neq 0$, with a left-invariant metric. For $\lambda>0, M(\lambda)$ has strictly negative curvature, constant for $\lambda=1$. Moreover, $M(\lambda)$ is a principal fiber bundle, over an $(n-1)$-dimensional space of constant negative curvature.

Proof. Let $\mathfrak{g}, \mathfrak{h}$ denote the Lie algebras of $G$ and $H$, respectively. Let $\mathfrak{m}$ be an ad(h)-invariant complement of $\mathfrak{h}$ in $\mathfrak{g}$, and $\mathfrak{m}=\mathfrak{m}_{1}+\mathfrak{\mathfrak { m }}$ as in (2.1). By (2.4) we have $\left[\tilde{\mathfrak{m}}, \mathfrak{m}_{1}\right] \cong \mathfrak{m}_{1}$. If $\left[\tilde{\mathfrak{m}}, \mathfrak{m}_{1}\right]=0$ then Theorem $\mathrm{A}$ implies case (1).

Assume then that $\left[\tilde{\mathfrak{m}}, \mathfrak{m}_{1}\right] \neq 0$, and let $\alpha \in \tilde{\mathfrak{m}}^{*}$ be as in $(2.7)$. Choose a unit vector $X \in \tilde{\mathfrak{m}}^{\prime}=\operatorname{Ker}(\alpha)$ and let $Y \in \tilde{\mathfrak{m}}$ be such that $\langle X, Y\rangle=0$ and $\alpha(Y)=1$. Up to scalar multiplication of the inner product $\langle$, $\rangle$, we can assume that $\|Y\|=1$. Notice that these choices determine a particular metric in each homothety class. By Proposition (2.9) we have that 


$$
[Y, X]=\lambda X, \quad \lambda \in \boldsymbol{R} .
$$

If $\lambda=0$, then the decomposition

$$
\mathfrak{g}=\left(\mathfrak{h}+\mathfrak{m}_{1}+\boldsymbol{R} Y\right) \oplus \boldsymbol{R} X
$$

is a direct sum of ideals and applying Lemma (3.4) we obtain case (2).

If $\lambda \neq 0$, then $\mathfrak{m}$ is a subalgebra of $\mathfrak{g}$ and hence by Lemma (3.3) $M$ is isometric to a Lie group $M(\lambda)$ with a left-invariant metric. The derived algebra $[\mathfrak{m}, \mathfrak{m}]=\mathfrak{m}_{1}+\boldsymbol{R} X$ is an abelian ideal of codimension 1 . Therefore $\mathfrak{m}$ is solvable and, moreover, we can apply Theorem 1 in [4] to conclude that if $\lambda>0 M(\lambda)$ has negative sectional curvature. The last statement in (3) follows from Remark (3.6).

(5.2) Proposition. The sectional curvatures of $M(\lambda)$ satisfy:

(i) For $\lambda>0, \min \left(-1,-\lambda^{2}\right) \leqq K \leqq \max \left(-1,-\lambda^{2}\right)$

(ii) For $\lambda<0, \min \left(-1,-\lambda^{2}\right) \leqq K \leqq-\lambda$.

Proof. The Lie algebra $\mathfrak{m}(\lambda)$ decomposes as

$$
\mathfrak{m}(\lambda)=\mathfrak{m}_{1}+\boldsymbol{R} X+\boldsymbol{R} Y
$$

with $\alpha(Y)=1$ and $[Y, X]=\lambda X$. It is enough to consider 2-dimensional subspaces of $\mathfrak{m}(\lambda)$ of the form

$$
\mathfrak{p}=\operatorname{span}_{R}\left\{a Y+b Z_{1}, Z_{2}\right\}
$$

where $Z_{i} \in \mathfrak{m}_{1}+\boldsymbol{R} X, i=1,2$, are orthonormal and $a^{2}+b^{2}=1$. We then have [4]

$$
K(\mathfrak{p})=-a^{2}\left\langle T^{2} Z_{2}, Z_{2}\right\rangle+b^{2}\left(\left\langle T Z_{1}, Z_{2}\right\rangle^{2}-\left\langle T Z_{1}, Z_{1}\right\rangle\left\langle T Z_{2}, Z_{2}\right\rangle\right)
$$

where $T=\operatorname{ad}(Y): \mathfrak{m}_{1}+\boldsymbol{R} X \rightarrow \mathfrak{m}_{1}+\boldsymbol{R} X$. If we now write

$$
Z_{i}=Z_{i}^{\prime}+z_{i} X, \quad Z_{i}^{\prime} \in \mathfrak{m}_{1}, \quad z_{i} \in \boldsymbol{R},
$$

then $T Z_{i}=Z_{i}+(\lambda-1) z_{i} X$ and (5.3) becomes

$$
\begin{aligned}
K(\mathfrak{p})=-a^{2}\left\langle Z_{2}+\left(\lambda^{2}-1\right) z_{2} X, Z_{2}\right\rangle^{2}+b^{2}\left(\left\langle Z_{1}+(\lambda-1) z_{1} X, Z_{2}\right\rangle^{2}\right. \\
\left.\quad-\left\langle Z_{1}+(\lambda-1) z_{1} X, Z_{1}\right\rangle\left\langle Z_{2}+(\lambda-1) z_{2} X, Z_{2}\right\rangle\right) \\
=-1-\left[\left(a^{2}\left(\lambda^{2}-1\right)+b^{2}(\lambda-1)\right) z_{2}^{2}+b^{2}(\lambda-1) z_{1}^{2}\right] .
\end{aligned}
$$

It is then clear that for $\lambda=1, K \equiv-1$. If $\lambda>1$ we have $K(\mathfrak{p}) \leqq-1$; on the other hand the expression between brackets attains its maximum for $z_{1}=0$, $z_{2}=1, a=1, b=0$ and thus $-\lambda^{2} \leqq K(\mathfrak{p}) \leqq-1$. Similarly, if $0<\lambda<1,-1 \leqq K(\mathfrak{p})$ and the maximum of $K(\mathfrak{p})$ is attained at the same point giving $-1 \leqq K(\mathfrak{p}) \leqq-\lambda^{2}$. This proves (i); an analogous argument shows (ii).

(5.4) Corollary. If $\lambda_{1} \neq \lambda_{2}$ then $M\left(\lambda_{1}\right)$ is not homothetic to $M\left(\lambda_{2}\right)$.

The spaces $M(\lambda), \lambda>0$, constitute therefore a "one-parameter" family of solvable Lie groups admitting a left-invariant metric of strictly negative 
curvature. These spaces have been studied by Heintze [4] and by Azencott and Wilson [1], [2], who have given an infinitesimal characterization of the full isometry group of such a solvmanifold. In our case we have

(5.5) Theorem. Let $M(\lambda)=G / H, 0<\lambda \neq 1$, be as in (3) of Theorem (5.1). Then $G \cong I_{0}(M(\lambda))$.

PRoof. If $\operatorname{dim} I_{0}(M(\lambda))>\operatorname{dim} G$, then the isotropy subgroup of $I_{0}(M(\lambda))$ at the origin $o=\{H\} \in M(\lambda)$ must be isomorphic to one of the following: $S O(n)$, $S O(n-1)$ or $S O(n-2) \times S O(2)$. Since for $\lambda \neq 1, M(\lambda)$ is not a space of constant curvature it is clear that the $S O(n)$-case cannot occur. In either of the remaining two cases $M(\lambda)$ would have a Euclidean factor which is impossible since $M(\lambda)$ has strictly negative curvature.

We shall next consider the case $H=S O(n-3) \times K$. If $K \cong S O(3)$ then Theorem $C$ implies that $M \cong M_{1}^{(n-3)} \times M_{2}^{(3)}$, where $M_{i}$ is a simply-connected space of constant curvature and $G \cong I_{0}\left(M_{1}\right) \times I_{0}\left(M_{2}\right)$. If $K=\{e\}$ and $n-3 \geqq 3$, then we may apply Theorem A to conclude that either $M$ is isometric to a product $M \cong M_{1}^{(n-3)} \times M_{2}^{(3)}$, where $M_{1}$ is an $(n-3)$-dimensional space of constant curvature and $M_{2}$ is a 3-dimensional simply-connected Lie group with a leftinvariant metric, (For a classification of these Lie groups together with their curvature properties, relative to a left-invariant metric, we refer to Milnor [10]), or $M$ is itself isometric to a Lie group with a left-invariant metric. We recall how this latter case arises: Let

$$
\mathfrak{g}=\mathfrak{h}+\mathfrak{m}_{1}+\tilde{\mathfrak{m}}
$$

be as in (2.1), and assume $\tilde{\mathfrak{m}}^{\prime} \neq \tilde{\mathfrak{m}}$. By (2.11) we then have that $\mathfrak{m}_{1}$ is an abelian ideal in g. As before let $Y \in \tilde{\mathfrak{m}}$, be a unit vector, orthogonal to $\tilde{\mathfrak{m}}^{\prime}$ and such that $\alpha(Y)=1$. The study of the Lie algebra $\mathfrak{m}$ (and thus of $\mathfrak{g}$ ) now reduces to the study of the 3-dimensional sub-algebra $\mathfrak{m}$. We consider the following cases :

(i) $[\tilde{\mathfrak{m}}, \tilde{\mathfrak{m}}]=0$. In particular the decomposition

$$
\mathfrak{g}=\left(\mathfrak{h}+\mathfrak{m}_{1}+R Y\right) \oplus \tilde{\mathfrak{m}}^{\prime}
$$

is a direct sum of ideals and therefore $M$ is isometric to a product $M \cong M_{1}^{(n-2)} \times \boldsymbol{R}^{2}$ where $M_{1}$ is an $(n-2)$-dimensional space of constant negative curvature.

(ii) $[\tilde{\mathfrak{m}}, \tilde{\mathfrak{m}}]=\tilde{\mathfrak{m}}^{\prime}$, i. e. $\operatorname{dim}[\tilde{\mathfrak{m}}, \tilde{\mathfrak{m}}]=2$. We first prove

(5.6) LEMMA. $\tilde{\mathfrak{m}}^{\prime}$ is an abelian ideal.

ProOF. Let $X_{1}, X_{2}$ be a basis of $\tilde{\mathfrak{m}}^{\prime}$ such that

$$
\left[X_{1}, X_{2}\right]=\lambda X_{2} \text {. }
$$

Let $\left[Y, X_{i}\right]=\sum_{j=1}^{2} a_{i j} X_{j}, i=1,2$. Then 


$$
\lambda\left[Y, X_{2}\right]=\left[Y,\left[X_{1}, X_{2}\right]\right]=\left[\left[Y, X_{1}\right], X_{2}\right]+\left[X_{1},\left[Y, X_{2}\right]\right]
$$

and we have

$$
\lambda \sum_{j=1}^{2} a_{2 j} X_{j}=\lambda a_{11} X_{2}+\lambda a_{22} X_{2}
$$

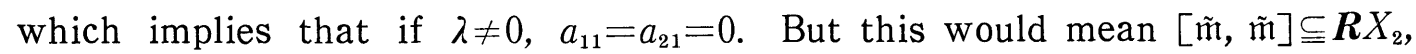
contradicting assumption (ii). Hence $\lambda=0$ and (5.6) is proved.

It is clear now that in this case the derived algebra $[\mathfrak{m}, \mathfrak{m}]=\mathfrak{m}_{1}+\tilde{\mathfrak{m}}^{\prime}$ is an abelian ideal of codimension 1 . We can therefore apply Theorem 1 in [4] to conclude

(5.7) PROPOSITION. Let D (respectively S) denote the symmetric (respectively skew-symmetric) part of the linear transformation

$$
\operatorname{ad}(Y): \tilde{\mathfrak{m}}^{\prime} \rightarrow \tilde{\mathfrak{m}}^{\prime} .
$$

Then $M$ admits a left-invariant metric with strictly negative curvature if and only if

(a) $D$ is positive definite

(b) $D^{2}-D S-S D$ is positive definite.

If in addition we assume that $\tilde{\mathfrak{m}}$ is unimodular (i.e. $\operatorname{tr}(\operatorname{ad} Y)=0$ ) then a straightforward argument shows that there exists a basis $X_{1}, X_{2}$ of $\tilde{\mathfrak{m}}^{\prime}$ such that $\left[Y, X_{i}\right]=\mu X_{j}, i \neq j, \mu \neq 0$. Therefore $\tilde{\mathfrak{m}} \cong E(1,1)$, the Lie algebra of the group of rigid motions of Minkowski 2-space [10]. Moreover it follows from (5.7) that $M$ does not admit a left-invariant metric with strictly negative curvature.

On the other hand if $\tilde{\mathfrak{m}}$ is not unimodular then $\operatorname{tr}(\operatorname{ad} Y)$ and $\operatorname{det}(\operatorname{ad} Y)$ are a complete set of isomorphism invariants for the Lie algebra $\mathfrak{m}[10]$. In this case the ideal $\tilde{\mathfrak{m}}^{\prime}$ may be characterized as the unimodular kernel of $\tilde{\mathfrak{m}}$.

(iii) $\operatorname{dim}[\tilde{\mathfrak{m}}, \tilde{\mathfrak{m}}]=1$. In this case $[\mathfrak{m}, \mathfrak{m}]$ is an abelian ideal of codimension 2 and [4, Proposition 2] implies that $M$ does not admit a left-invariant metric with strictly negative curvature. Moreover, $\tilde{m}$ is not unimodular and the trace of $\operatorname{ad}(Y)$ acting on the unimodular kernel of $\tilde{\mathfrak{m}}$ is a complete isomorphism invariant for $\mathfrak{m}$.

Now we consider the case $M=G / H, H \cong S O(n-3) \times S O(2), n-3 \geqq 3$. In this case $\operatorname{dim} \tilde{\mathrm{m}}=1$ and $\mathrm{g}$ decomposes according to (3.1) as

$$
\begin{aligned}
& \mathfrak{g}=\mathfrak{h}_{1}+\mathfrak{h}_{2}+\mathfrak{m}_{1}+\mathfrak{m}_{2}+\tilde{\mathfrak{m}}, \\
& \mathfrak{h}_{1} \cong \operatorname{so}(n-3), \quad \mathfrak{h}_{2} \cong s o(2) .
\end{aligned}
$$

We set $\tilde{\mathfrak{m}}_{1}=\mathfrak{h}_{2}+\mathfrak{m}_{2}+\tilde{\mathfrak{m}}$. We have

$$
\left[\mathfrak{h}_{1}, \tilde{\mathfrak{m}}_{1}\right]=0, \quad\left[\tilde{\mathfrak{m}}_{1}, \mathfrak{m}_{1}\right] \leqq \mathfrak{m}_{1} .
$$

Let $\tilde{\mathfrak{m}}_{1}^{\prime}=\left\{X \in \tilde{\mathfrak{n}}_{1}:\left[X, \mathfrak{m}_{1}\right]=0\right\}$. If $\tilde{\mathfrak{n}}_{1}=\tilde{\mathfrak{n}}_{1}^{\prime}$ then $a$ is a split Lie algebra 
$\mathfrak{g}=\mathfrak{g}_{1} \oplus \mathfrak{g}_{2}$ where

$$
\begin{aligned}
& \mathfrak{g}_{1}=\mathfrak{h}_{1}+\mathfrak{m}_{1} \\
& \mathfrak{g}_{2}=\mathfrak{h}_{2}+\mathfrak{m}_{2}+\tilde{\mathfrak{m}} .
\end{aligned}
$$

It then follows from (3.4) that

(5.8) $M$ is isometric to a product

$$
M \cong M_{1}^{(n-3)} \times M_{2}^{(3)}
$$

where $M_{1}$ is a simply-connected space of constant curvature and $M_{2}$ is a 3-dimensional simply-connected manifold admitting a 4-dimensional transitive group of isometries. These spaces have been classified in (4.11).

Assume now $\tilde{\mathfrak{m}}_{1}^{\prime} \neq \tilde{\mathfrak{m}}_{1}$. Then $\operatorname{dim} \tilde{\mathfrak{m}}_{1}^{\prime}=3$. Moreover, $\mathfrak{h}_{2} \subseteq \tilde{\mathfrak{m}}_{1}^{\prime}$ and since $\tilde{\mathfrak{m}}_{1}^{\prime}$ is an ideal, $\mathfrak{m}_{2}=\left[\mathfrak{h}_{2}, \mathfrak{m}_{2}\right] \subseteq \tilde{\mathfrak{m}}_{1}^{\prime}$. Hence

$$
\tilde{\mathfrak{m}}_{1}^{\prime}=\mathfrak{h}_{2}+\mathfrak{m}_{2} \text {. }
$$

Let $Y$ be a unit vector in ${ }^{\circ}$ such that ad $Y: \mathfrak{m}_{1} \rightarrow \mathfrak{m}_{1}$ is the identity map (again this is possible up to homothety). Furthermore, as in Section 4 we can also assume (changing $\mathfrak{m}_{2}$ if necessary) that there exists $\lambda \in \boldsymbol{R}$ such that

$$
[Y, X]=\lambda X, \quad X \in \mathfrak{m}_{2} .
$$

We have also shown in Section 4 that $\left[\mathfrak{m}_{2}, \mathfrak{m}_{2}\right] \subseteq \tilde{\mathfrak{m}}$. Therefore if $E_{1}, E_{2}$ is a basis of $\mathfrak{m}_{2}$ we have

$$
\left[E_{1}, E_{2}\right]=\alpha Y, \quad \alpha \in \boldsymbol{R} .
$$

But if $0 \neq Z \in \mathfrak{m}_{1}$, we get

$$
0=\left[\left[E_{1}, E_{2}\right], Z\right]=\alpha[Y, Z]=\alpha Z .
$$

Consequently $\left[\mathfrak{m}_{2}, \mathfrak{m}_{2}\right]=0$, the subalgebra $\mathfrak{m}$ is solvable and the derived algebra $[\mathfrak{m}, \mathfrak{m}]=\mathfrak{m}_{1}+\mathfrak{m}_{2}$ is an abelian ideal of codimension 1 .

If $\lambda=0$ then $g$ splits as $\mathfrak{g}=\mathfrak{g}_{1} \oplus \mathfrak{g}_{2}$, with

and therefore

$$
\mathfrak{g}_{1}=\mathfrak{h}_{1}+\mathfrak{m}_{1}+R Y, \quad \mathfrak{g}_{2}=\mathfrak{h}_{2}+\mathfrak{m}_{2}
$$

(5.9) $M$ is isometric to a product $M_{1}^{(n-2)} \times \boldsymbol{R}^{2}$, where $M_{1}$ is a space of constant negative curvature. Moreover $G \cong G_{1} \times E(2)$, where $G_{1}$ is the group described in [6, Theorem 3.3] and $E(2)$ is the group of motions of Euclidean 2-space.

For $\lambda \neq 0, M$ is isometric to a solvable Lie group $M(\lambda)$ with a left-invariant metric and it follows from [4, Theorem 1] that

(5.10) If $\lambda>0, M(\lambda)$ has strictly negative curvature; for $\lambda<0, M(\lambda)$ has both positive and negative sectional curvatures.

It is straightforward to check that (5.2), (5.4) and (5.5) carry over to this case without modifications. 
Finally, we consider the case of a 5-dimensional homogeneous Riemannian manifold $M=G / H$ where $H \cong S O(2) \times S O(2)$ and the linear isotropy representation of $H$ is equivalent to $\rho_{2} \oplus \rho_{2} \oplus \theta_{1}$. As in (3.1) we can write

$$
\mathfrak{g}=\mathfrak{h}_{1}+\mathfrak{h}_{2}+\mathfrak{m}_{1}+\mathfrak{m}_{2}+\tilde{\mathfrak{m}} \text {. }
$$

Let $\tilde{\mathfrak{m}}_{1}=\mathfrak{h}_{2}+\mathfrak{m}_{2}+\tilde{\mathfrak{m}}, \tilde{\mathfrak{m}}_{2}=\mathfrak{h}_{1}+\mathfrak{m}_{1}+\tilde{\mathfrak{m}}$; then $\left[\mathfrak{h}_{i}, \tilde{\mathfrak{m}}_{i}\right]=0,\left[\tilde{\mathfrak{m}}_{i}, \mathfrak{m}_{i}\right] \leqq \mathfrak{m}_{i}$ and $\left[\tilde{\mathfrak{m}}_{i}, \tilde{\mathfrak{m}}_{i}\right]$ $\subseteq \tilde{\mathfrak{m}}_{i}^{\prime}=\left\{X \in \tilde{\mathfrak{m}}_{i}:\left[X, \mathfrak{m}_{i}\right]=0\right\}$.

If $\tilde{\mathfrak{m}}_{1} \neq \tilde{\mathfrak{m}}_{1}^{\prime}$, then $\mathfrak{g}_{2}=\mathfrak{h}_{2}+\mathfrak{m}_{1}+\mathfrak{m}_{2}+\tilde{\mathfrak{m}}$ is a subalgebra of $\mathfrak{g}$ and the corresponding analytic subgroup $G_{2} \cong G$ acts transitively on $M$ with isotropy $H_{2} \cong S O(2)$. Hence this case reduces to the one studied in Section 4.

We can therefore assume that $\tilde{\mathfrak{m}}_{i}=\tilde{\mathfrak{m}}_{i}^{\prime}$ for $i=1,2$. In particular we have

$$
\begin{gathered}
{\left[\mathfrak{m}_{1}, \mathfrak{m}_{2}\right]=\left[\mathfrak{m}_{1}, \tilde{\mathfrak{m}}\right]=\left[\mathfrak{m}_{2}, \tilde{\mathfrak{m}}\right]=0,} \\
{\left[\mathfrak{m}_{i}, \mathfrak{m}_{i}\right] \cong \mathfrak{h}_{i}+\tilde{\mathfrak{m}} .}
\end{gathered}
$$

Thus $\tilde{\mathfrak{m}}$ is an ideal in $\mathfrak{g}$ and the quotient $\mathfrak{g} / \tilde{\mathfrak{m}}$ is a split Lie algebra

$$
\mathfrak{g} / \tilde{\mathfrak{m}} \cong \mathfrak{g}_{1} \oplus \mathfrak{g}_{2}, \quad \mathfrak{g}_{i}=\mathfrak{h}_{i}+\mathfrak{m}_{i}+\tilde{\mathfrak{m}} / \tilde{\mathfrak{m}} .
$$

Moreover, the one-parameter group exp m̃ acts freely on $M$ and hence $M$ is a principal fiber bundle over $M / \exp \tilde{\mathfrak{m}}$. It is easy to check that, relative to the induced metric, the space splits isometrically as $M_{1} \times M_{2}$ where $M_{i}, i=1,2$ is a simply-connected 2-dimensional space of constant curvature.

\section{References}

[1] Robert Azencott and Edward N. Wilson, Homogeneous manifolds with negative curvature, I, Trans, Amer. Math. Soc., 215 (1976), 323-362.

[2] Robert Azencott and Edward N. Wilson, Homogeneous manifolds with negative curvature, II, Mem, Amer. Math. Soc., 8, no. 178 (1976).

[3] E. Cartan, Leçons sur la Géométrie des Espaces de Riemann, Gauthier-Villars, Paris, 1963.

[4] Ernst Heintze, On homogeneous manifolds of negative curvature, Math. Ann., 211 (1974), 23-34.

[5] Wu-chung Hsiang and Wu-yi Hsiang, Differentiable actions of compact connected classical groups I, Amer. J. Math., 89 (1967), 705-786.

[6] S. Kobayashi, Transformation Groups in Differential Geometry, Springer-Verlag, 1972.

[7] S. Kobayashi and T. Nagano, Riemannian manifolds with abundant isometries, Differential Geometry in Honor of K. Yano, Kinokuniya, Tokyo, 1972, 195-220.

[8] Minoru Kurita, On the isometry of a homogeneous Riemann Space, Tensor, 3 (1954), 91-100.

[9] Gordon W. Lukesh, Compact homogeneous Riemannian manifolds, Geometriae Dedicata, 7 (1978), 131-137.

[10] J. Milnor, Curvatures of left invariant metrics on Lie groups, Advances in Math., 21 (1976), 293-329. 
[11] H. Wakakuwa, On $n$-dimensional Riemannian spaces admitting some groups of motions of order less than $1 / 2 n(n-1)$, Tôhoku Math. J., 6 (1954), 121-134.

\section{E. H. Cattani}

Department of Mathematics University of Massachusetts Amherst, 01003

U.S.A.
L. N. MANN

Department of Mathematics University of Massachusetts Amherst, 01003

U.S.A. 